\title{
Pseudomorphic growth induced by chemical adatom potential
}

\author{
Karsten Bromann, Harald Brune *, Marcella Giovannini, Klaus Kern \\ Institut de Physique Expérimentale, EPF Lausanne, CH-1015 Lausanne, Switzerland
}

Received 5 May 1997; accepted for publication 16 June 1997

\begin{abstract}
The transformation from pseudomorphic to dislocated and back to pseudomorphic growth with increasing coverage is reported for molecular beam epitaxy of $\mathrm{Ag}$ on $\mathrm{Pt}(111)$. Below a critical size of $200 \AA \AA$ two-dimensional Ag islands grow coherently strained, while larger islands relieve strain through the introduction of misfit dislocations. Upon completion of the first monolayer, the dislocations disappear and the $\mathrm{Ag}$ film again adopts a pseudomorphic structure. With the help of effective-medium theory calculations, it is shown that this effect is related to the elevated chemical potential of $\mathrm{Ag}$ adatoms on top of the first $\mathrm{Ag}$ monolayer. $\mathrm{C} 1997$ Elsevier Science B.V.
\end{abstract}

Keywords: Dislocations; Elastic continuum theory; Epitaxy; Platinum; Scanning tunneling microscopy; Silver; Strain

The structure of ultrathin films on substrates of a different material is determined by the atomic interactions at the interface and the structures of the two respective bulk phases. A lattice mismatch between the film and the substrate material leads to strain in the film until the overgrowth has adopted its bulk geometry through the introduction of strain-relieving defects. Frank and van der Merwe [1] were the first to address theoretically strain relaxation in a heteroepitaxial system, which they modeled using the Frenkel-Kontorova approach [2] of a one-dimensional chain of atoms coupled by elastic springs in a sinusoidal (substrate) potential. Later, two- and three-dimensional models based on elastic continuum theory were developed [3-5]. These "continuum" models predict either a critical misfit above which dislocations form in a complete monolayer, or vice versa,

\footnotetext{
* Corresponding author. Fax: $(+41) 216933604$ e-mail: harald brune (a)ipe.dp.epfl.ch
}

a maximum film thickness for pseudomorphic growth which depends on the actual misfit of the system and the elastic material parameters.

Though heteroepitaxial metal films were believed to be well described by these theories, recently strain relief mechanisms were revealed which lead to deviations from the predictions [6-11]. On hexagonal close-packed interfaces, for example, strain can easily be accommodated by fcc-hcp stacking faults, and the critical thickness is decreased to zero or just the first monolayer. These so-called partial dislocations can arrange in a variety of structures, including unidirectional or trigonal dislocation networks [7-9] or even dislocation loops in the substrate [10]. Further, strong relaxation effects in the substrate can stabilize such structures energetically [11]. The resulting morphologies in these systems often vary from layer to layer, and for each respective layer metastable configurations other than the energetically most favored sometimes exist. However, despite their 


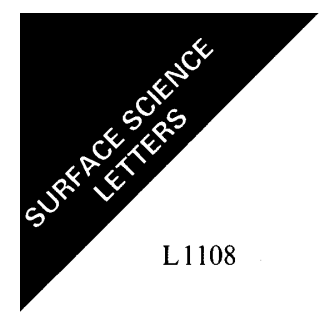

structural variety, all these systems have one feature in common, i.e the existence of a critical coverage above which, if not kinetically hindered, strain-relieving defect structures form until the growing film has adopted its bulk geometry.

Here we present an interesting deviation from this common behavior of heteroepitaxial metal systems. In our example of the first monolayer of $\mathrm{Ag}$ on $\mathrm{Pt}(111)$, a dislocation network evolves when monolayer islands reach a critical size and thereby accumulate a critical amount of strain. Surprisingly, this dislocation network disappears completely upon completion of the first monolayer and the initial growth of the second monolayer. We demonstrate that the maximum lateral size for pseudomorphic growth of two-dimensional islands is determined by the delicate balance between edge relaxation and dislocation formation. The re-entrant pseudomorphic growth upon monolayer completion is explained by taking into account the chemical potential of an adatom gas present on top of the adlayer.

So far, experiments performed with STM, He diffraction, LEED and photoelectron diffraction (PED) reported the first monolayer of $\mathrm{Ag}$ to grow pseudomorphically on $\operatorname{Pt}(111)[8,12-14]$. With increased resolution, however, STM reveals a network of double lines, imaged with a depth of less than $0.1 \AA$, running along the three $\langle 11 \overline{2}\rangle$ directions perpendicular to the close-packed atom rows, as demonstrated in Fig. 1 [15]. The experiments were performed with a variable-temperature STM based in a UHV chamber described in detail elsewhere [16]. Ag was deposited from an $\mathrm{MBE}$ Knudsen cell at rates of $1 \mathrm{ML}$ per $900 \mathrm{~s}$. In analogy to similar structures in the literature [7-9,11], we infer that the double lines in Fig. 1 are two partial dislocations, forming "light" domain walls between fcc and hcp stacking, as $\mathrm{Ag}$ on $\mathrm{Pt}(111)$ is compressively strained by $4.3 \%$. With PED it was found that the first monolayer of $\mathrm{Ag}$ grows in fcc stacking to the substrate. From this and the fact that the dislocations were never found to end at ascending substrate steps, we conclude that the small regions within the double lines have hcp stacking. This is further corroborated by a recent density functional calculation which revealed that fcc stacking is favored by $\sim 30 \mathrm{meV}$ per Ag atom
[17]. Where the structure is well ordered over a larger region, the double lines have an average spacing of $75 \AA$, corresponding to $26 \mathrm{Ag}$ atoms on $27 \mathrm{Pt}$ atoms in the close-packed direction perpendicular to the dislocations. This periodicity agrees well with a simplistic geometric picture where one would expect a $\sqrt{3} \times 24$ unit cell for complete unidirectional strain relief. Note, however, that the mesoscopic order of the dislocations is very sensitive to defects. Vacancies in the Ag film act as preferential ending points for the dislocations and can cause local variations from their average spacing. They further prevent the formation of large rotational domains.

We followed the dislocation formation as a function of island size and coverage. At $300 \mathrm{~K}, \mathrm{Ag}$ is sufficiently mobile on the $\operatorname{Pt}(111)$ surface that film growth, even on very large terraces, proceeds from substrate step edges. In order to study the influence of island size, we prepared well-defined two-dimensional Ag islands by depositing a submonolayer coverage of $10 \%$ at $50 \mathrm{~K}$, where $\mathrm{Ag}$ diffusion is nearly frozen in, and subsequent annealing to $300 \mathrm{~K}$. In an Ostwald ripening process, compact islands form which are homogeneously distributed over the surface [18]. The average size of these islands can then be increased by further deposition of $\mathrm{Ag}$ at $300 \mathrm{~K}$. Fig. $2 \mathrm{a}$ shows that small islands grow pseudomorphically, whereas in larger islands dislocations have formed. The transition occurs gradually at island diameters of around $200 \AA$ (Fig. 2b). (We here refer to island diameter as the lateral extension of an island in the $\langle 1 \overline{1} 0\rangle$ direction, i.e. perpendicular to the dislocations if they are present.) It should be noted that this value is much larger than the average spacing of dislocations in a compact film. This indicates that for islands there must be an additional effective way for strain relief, which can only be at the edges. For small island sizes, the compressive strain can be relieved efficiently at the island edges through outward relaxation. At larger sizes, however, the accumulated strain is too large, and dislocations form.

The structures described so far are found in the whole submonolayer range. At coverages of around $1 \mathrm{ML}$, however, the dislocation network 


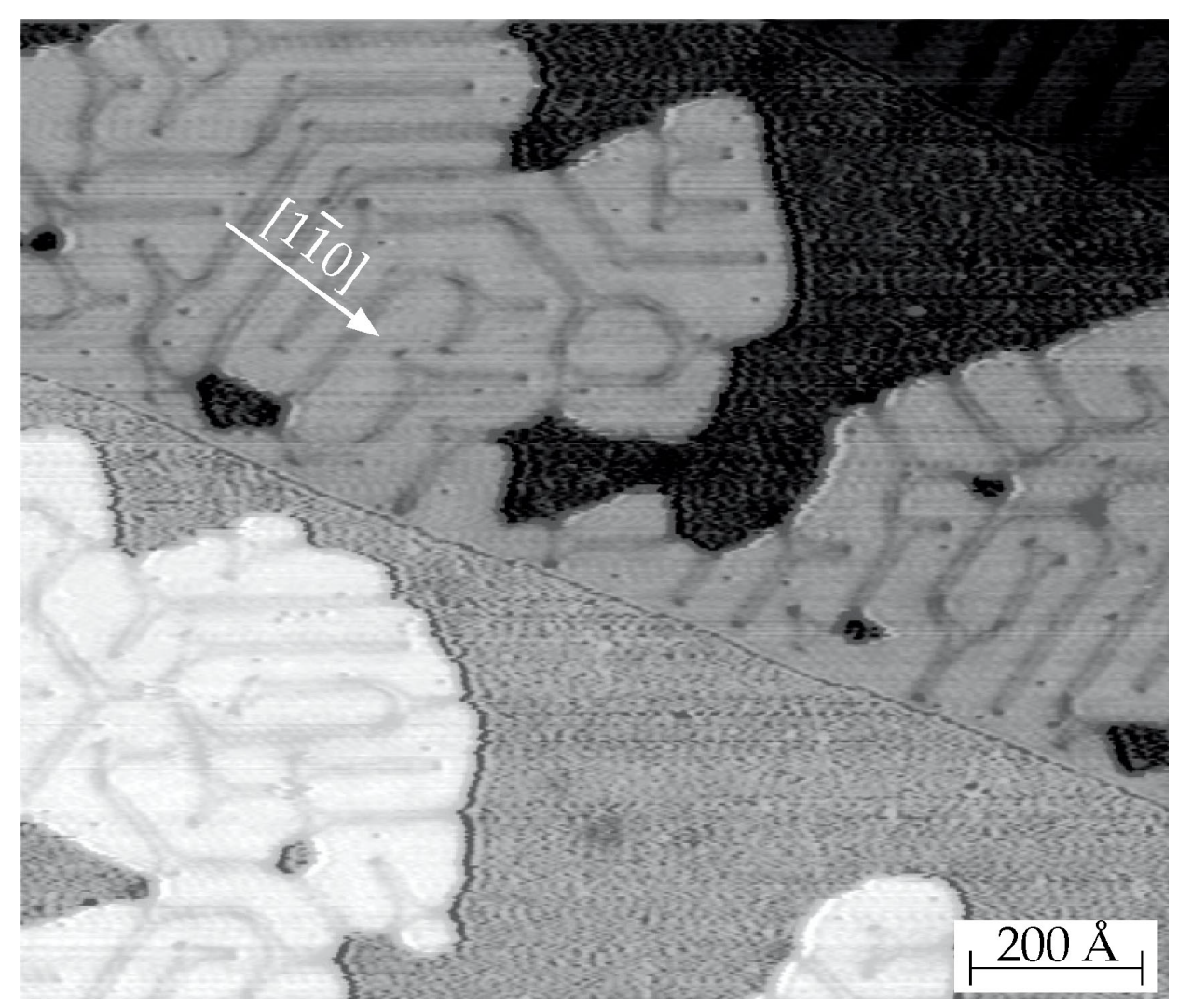

Fig. 1. The dislocation network in a first-ML Ag film growing from the steps of a $\mathrm{Pt}(111)$ substrate (coverage $=0.5 \mathrm{ML}$, deposition temperature $=500 \mathrm{~K}$ ).

in the first layer is lifted. Fig. 3a shows a $1.4 \mathrm{ML}$ film grown at $400 \mathrm{~K}$ after annealing to $800 \mathrm{~K}$ [19]. The equilibrium structure of the second $\mathrm{ML}$, a trigonal network of dislocation lines [8], can be easily distinguished from the first ML. Apart from a few defects induced by the direct proximity of the second ML, the first ML now is perfectly pseudomorphic. Further information about this re-entrant pseudomorphic growth can be gained from experiments in which second-ML islands are grown on an incomplete first-ML film. In such systems no dislocations are found in the first ML (Fig. 3b), which shows that it is not the completion of the first $\mathrm{ML}$ but rather the presence of the second ML which is the reason for the re-entrant pseudomorphic structure.

This finding leads to an explanation of the effect. With the presence of second-ML material, a 2D adatom gas on top of the $\mathrm{Ag}$ film is established which is in equilibrium with the condensed phase. This adatom gas, which is absent without second-ML material, can shift the equilibrium situation of the film. In a theoretical study, Needs et al. [20] argued that three energetic factors have to be considered in determining whether or not a surface is stable against the formation of dislocations. First, strain at an interface induces elastic energy in the film, which favors dislocation formation. Second, dislocations reduce the bonding energy to the substrate, as at least some of the atoms in the overlayer have to shift out of registry, which does not favor their formation. Third, the formation of dislocations changes the total number of atoms in the overlayer. The energy changes associated with the transfer of atoms into or out of the surface layer also have to be considered. If 


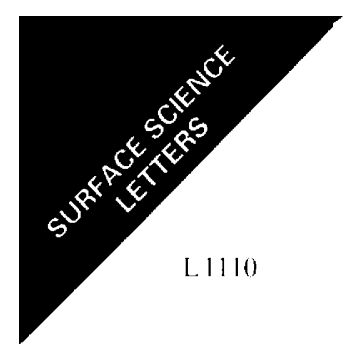

a)

b)
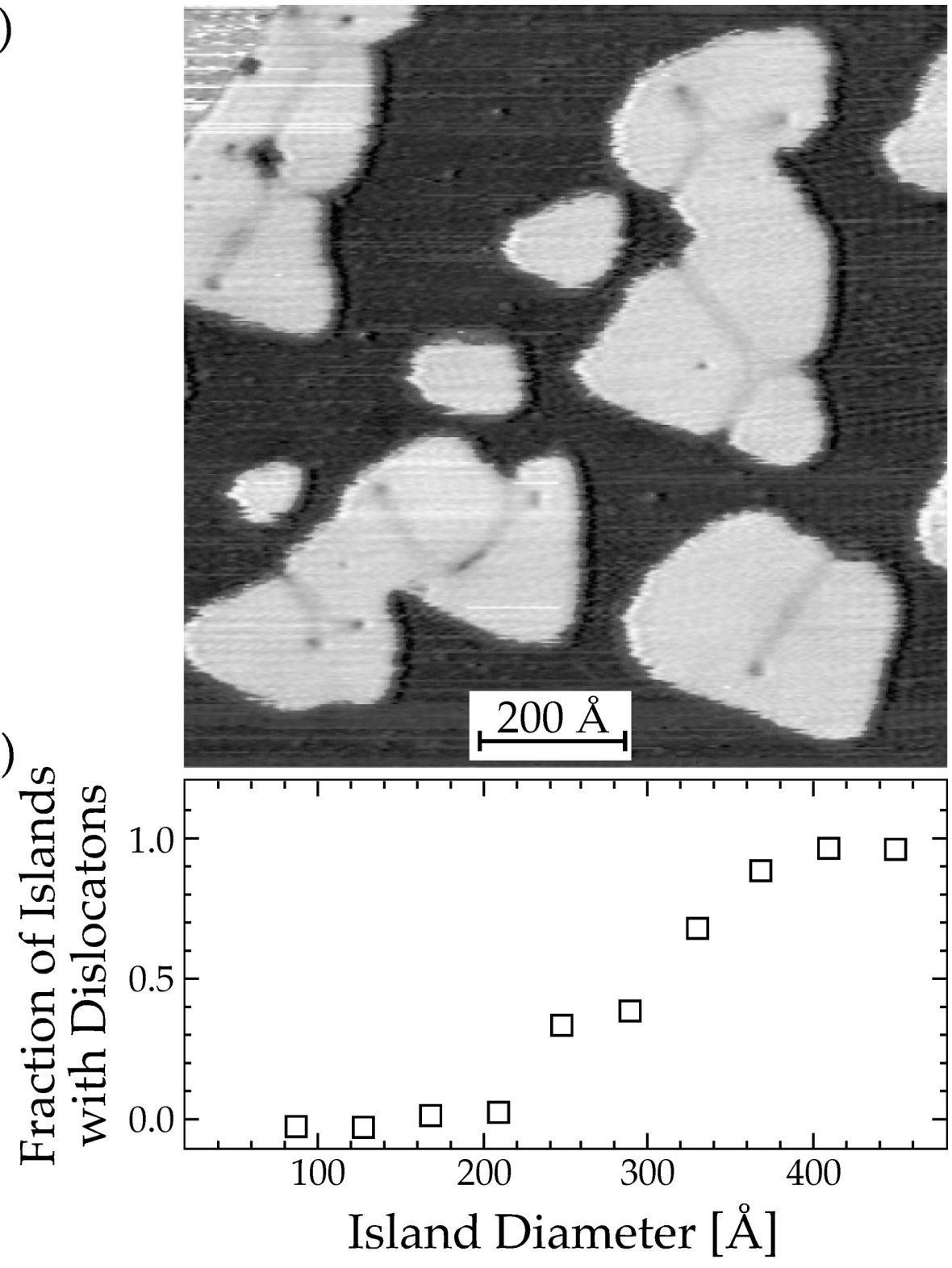

Fig. 2. (a) A population of $\mathrm{Ag}$ islands on $\mathrm{Pt}(111)$ grown by deposition of $0.12 \mathrm{ML} \mathrm{Ag}$ at $50 \mathrm{~K}$, subsequent Ostwald ripening, and post-evaporation of $0.25 \mathrm{ML}$ at $300 \mathrm{~K}$. (b) The probability of dislocation formation as a function of island size, as inferred from analysis of 180 islands.

the first two contributions are so close as to balance each other, the chemical potential of an adatom gas on top of the layer in question can hence be the determining factor. Evidently, it is this third aspect which is not accounted for in continuum theories of dislocation formation.
Recently, it was shown for Pt(111) homoepitaxy that increased chemical potential of an adatom gas can induce a reconstruction of the normally pseudomorphic surface [21]. In our system, under compressive strain, the inverse case is expected. Excess atoms from the adatom gas can be incorpo- 

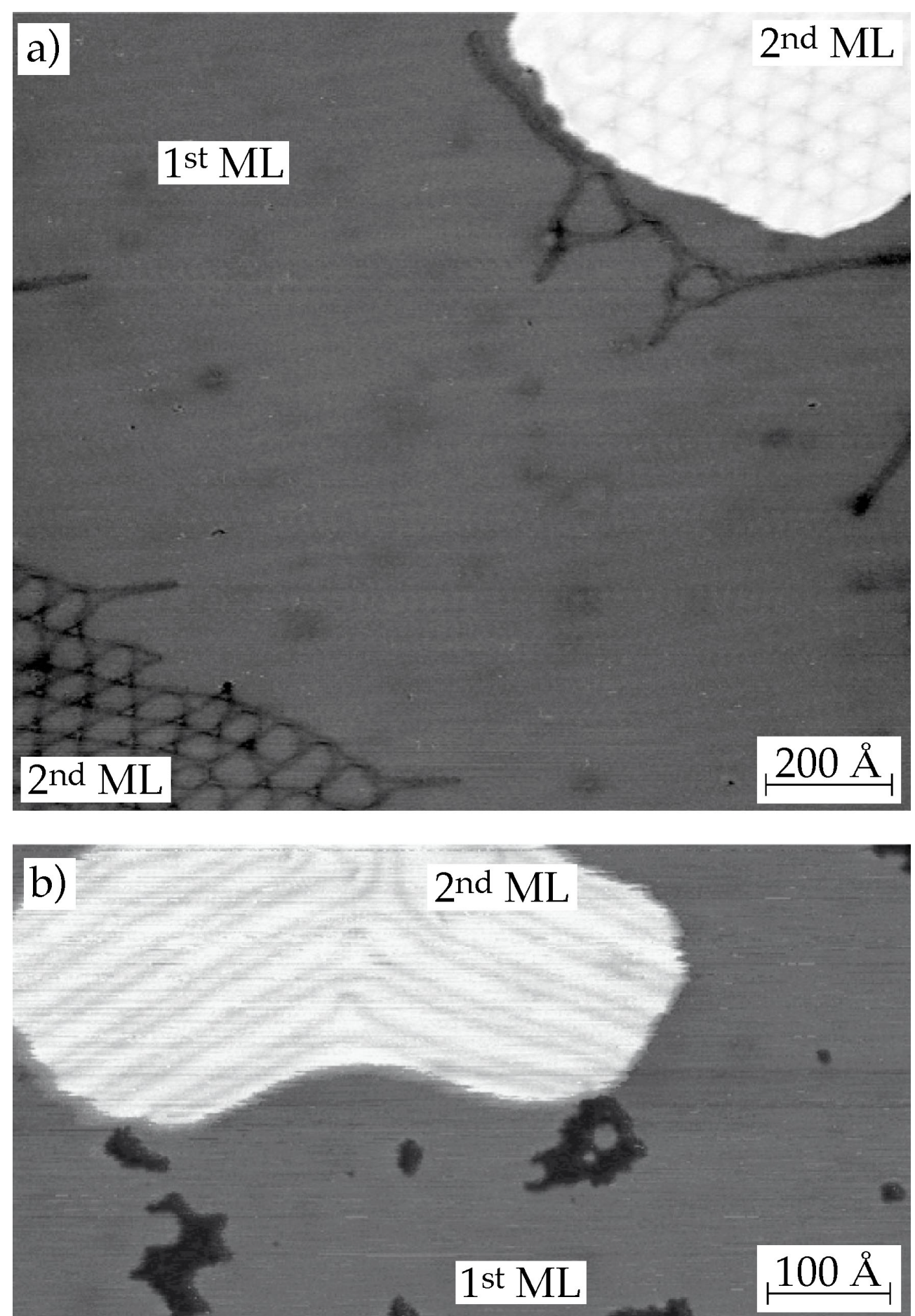

Fig. 3. Re-entrant pseudomorphic structure of the first ML in the presence of second-ML material. The dislocations disappear either upon annealing to $800 \mathrm{~K}$ and thus creating a first-ML film without vacancies (a), or on post-evaporation onto an as yet incomplete film at $300 \mathrm{~K}$ (b). 


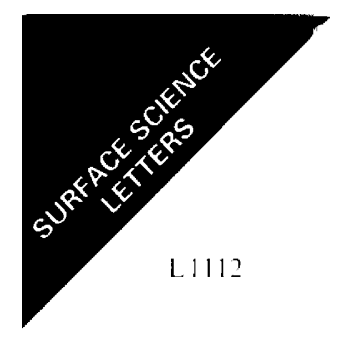

rated into the light walls and lift the dislocation network.

We performed effective-medium theory (EMT) calculation is [22] to support the above conclusions. To study $\mathrm{t}$ re strain-relief mechanism via relaxation at the edg:s, we calculated the atomic equilibrium positions $\mathrm{n}$ hexagonal $\mathrm{Ag}$ islands on a $\mathrm{Pt}(111)$ slab with island diameters ranging from 15 to $120 \AA$. In all cases, edge effects were found to be substantia. Independent of island size, the edge atoms were found to relax outwards by $\sim 2.5 \%$. The edge $r$ slaxation is distributed over 5-10 atomic rows, and the interatomic distances decay asymptotically fiom the edge towards the island core. Due to this very long-range effect, very small islands (br low $\sim 50 \AA$ in diameter) are essentially incommen iurate, whereas for larger islands a pseudomorphic core to the underlying $\mathrm{Pt}$ develops. With further increasing size the pseudomorphic core gains weight with respect to the relaxed edges, and finally the islands become unstable towards dislocation formation.

Calculations concerning dislocation formation in compacl films (or in the centers of large islands) were carried out with rectangular slabs of $3 \sqrt{3} \times N(V=10-50)$. The thickness of the slabs had to be chosen to comprise at least 20 layers, as strong relaxation effects influenced the subsurface Pt layers considerably to a depth of about 15 layers. To obtain the binding energy per $\mathrm{Ag}$ atom $\epsilon_{0}$ in a film with dislocations, we first placed a $3 \sqrt{3} \times(N-1)$ Ag layer (thus containing two partial dislocations) on top of a $3 \sqrt{3} \times N$ Pt substrate. The total energy of the fully relaxed slab is then subtracted from that of the substrate alone and divided by the number of $\mathrm{Ag}$ atoms. The same procedure is then repeated for a pseudomorphic Ag adlayer. Fig. 4 shows that as soon as $N>18$, dislocation formation is energetically favored. The structure with the highest $\mathrm{Ag}$ binding energy $\left(\epsilon_{0}=2.998 \mathrm{eV}\right.$ for $\left.N=30\right)$ lies $3 \mathrm{meV}$ per $\mathrm{Ag}$ atom below that of a pseudomorphic Ag layer. Hence, the energy gain per unit cell is $\sim 0.08 \mathrm{eV}$ if we take $N$ to be 27 , the experimentally determined average size of the superstructure. (A difference in total energy per atom of $3 \mathrm{meV}$ is certainly within the error of an approximate method such as EMT.

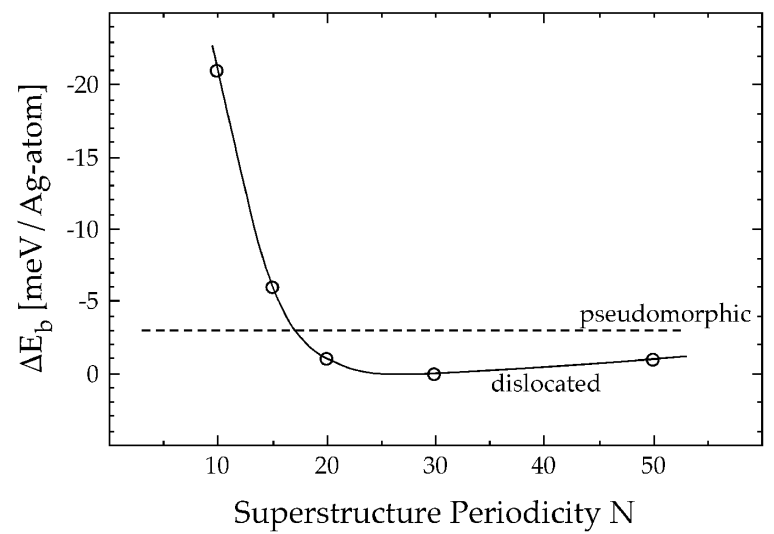

Fig. 4. Difference in binding energy per $\mathrm{Ag}$ atom between $\mathrm{Ag}$ adlayers of varying periodicity of the dislocation network and the ground state of the system $(\mathrm{a}(\sqrt{3} \times 29) \mathrm{Ag}$ adlayer with two partial dislocations on a $(\sqrt{3} \times 30) \mathrm{Pt}(111)$ substrate: binding energy per $\mathrm{Ag}$ atom $=2.998 \mathrm{eV}$ ). The binding energy of the pseudomorphic adlayer is given by the dotted line. As soon as $N>18$, dislocation formation is energetically favored.

However, the interesting aspect in our context is not that the calculations find the dislocated structure to be energetically favored, but rather the finding that the two structures are indeed very close to energetic degeneracy, which is the necessary condition for the possibility of the transition being driven by the second-layer adatom gas.) Note that EMT does not account for the energetic difference between fec and hcp sites found with DFT. This difference should drive the system even closer towards energetic degeneracy, and is responsible for the pair of partial dislocation lines running closely together in the real system, whereas they relax towards maximum separation in EMT simulations.

An isolated adatom on top of the first ML is found to be $0.62 \mathrm{eV}$ weaker bound than an atom embedded in the reconstructed first ML, as calculated with EMT. Consequently, if such adatoms are incorporated into the reconstructed adlayer and the dislocation network is lifted, the system gains about $0.62-0.08 \mathrm{eV}=0.54 \mathrm{eV}$ per $\sqrt{3} \times 27$ superstructure lattice cell. Thermodynamically, we can attribute a chemical potential of

$\mu_{\mathrm{g}}=\epsilon-k T \ln \left[\frac{A}{N} \frac{2 \pi m k T}{\hbar^{2}}\right]$ 


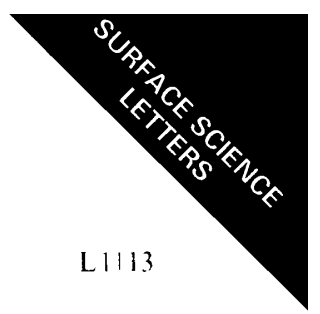

to the (ideal) 2D gas of silver atoms on top of the first-ML Ag film ( $\epsilon$ being the adsorption energy of the atoms, $m$ their mass, $\hbar$ Planck's constant and $N / A$ the density of the atoms). The free energy of the film, on the other hand, increases by $0.08 \mathrm{eV}$ per incorporated atom, i.e. $\mu_{\mathrm{f}}=0.08 \mathrm{eV}$. (Here we ignore entropy changes associated with the structural transformation of the film.) Equating chemical potentials for $T=300 \mathrm{~K}$ leads to a critical density of 1 atom per $3700 \AA^{2}$ at which Ag adatoms of the second ML should be incorporated into the first ML. This density can easily be maintained by adatoms evaporating from the edges of second-ML islands.

A closer look at the equilibrium positions of the atoms as determined by EMT shows that the absolute height of the $\mathrm{Ag}$ atoms in the domain walls, though sitting on bridge sites, is reduced. The depth of the indentations corresponds well to the measured height of the dislocations, showing that not electronic effects but pure geometry is sufficient to explain the imaging of STM. As suggested by Hwang et al. [11], the reason for this behavior lies in a very strong relaxation of the underlying substrate layers. Our EMT calculations reveal that below the dislocations, the first and second Pt layers are depressed by 0.14 and $0.15 \AA$, respectively. Though the relative distance between the first Pt layer and the Ag atoms on bridge sites is indeed increased, the absolute heights of the latter are reduced.

While we have observed re-entrant pseudomorphic growth for a particular model system, its implications for heteroepitaxial growth are of general significance. It is useful to distinguish systems under tensile and compressive strain. In the latter case, strained epitaxial islands tend initially to grow dislocation-free. The compressive strain can be relieved efficiently through outward relaxation at island edges, favoring relaxed growth for very small islands followed by pseudomorphic growth up to a critical size. However, as islands increase in size, the introduction of misfit dislocations will finally allow for a better elastic relaxation of the islands' stress. Dislocation formation in compressively strained layers can, however, be suppressed by the application of an enhanced gas-phase chemical potential if the energy gain due to strain relief and the energetic cost of dislocation formation are close to balancing each other. High supersaturation might thus be used to grow pseudomorphic structures which are otherwise unstable.

In systems with tensile strain, the effect of an enhanced gas-phase potential is inverse. Here, the presence of an adatom gas can drive an otherwise pseudomorphic system towards dislocation formation. Examples are the $\mathrm{Pt}(111)$ reconstruction during homoepitaxial growth [21] or the Ni monolayer structure on $\mathrm{Ru}(0001)$ [23], which is pseudomorphic at submonolayer coverages but becomes dislocated with the build-up of the second Ni layer. However, the effect of an enhanced adatom chemical potential favoring dislocation formation makes it impossible in principle to find re-entrant pseudomorphic behavior in systems under tensile stress.

\section{Acknowledgements}

The authors wish to thank Jens Nørskov and his group for allowing us to use their EMT computer code.

\section{References}

[1] F.C. Frank, J.H.v.d. Merwe, Proc. R. Soc. London A 198 (1949) 205.

[2] J. Frenkel, T. Kontorova, Zh. Eksp. Teor. Fiz. 8 (1939) 1340.

[3] W.A. Jesser, D. Kuhlmann-Wilsdorf, Phys. Status Solidi 19 (1967) 95

[4] J.W. Matthews, A.E. Blakeslee, J. Crystal Growth 27 (1974) 118.

[5] J.H.v.d. Merwe, C.A.B. Ball, in: Epitaxial Growth, Material Science Series, Academic Press, London, 1975, p. 493.

[6] B. Müller, B. Fischer, L. Nedelmann, A. Fricke, K. Kern, Phys. Rev. Lett. 76 ( 1996) 2358.

[7] G.O. Pötschke, R.J. Behm, Phys. Rev. B 44 (1991) 1442.

[8] H. Brune, H. Röder, C. Boragno, K. Kern, Phys. Rev. B 49 (1994) 2997.

[9] C. Günther, J. Vrijmoeth, R.Q. Hwang, R.J. Behm, Phys. Rev. Lett. 74 ( 1995 ) 754.

[10] J. Jacobsen, L.P. Nielsen, F. Besenbacher, I. Stensgaard, E. Lægsgaard, T. Rasmussen, K.W. Jacobsen, J.K. Nørskov, Phys. Rev. Lett. 75 (1995) 489.

[11] R.Q. Hwang, J.C. Hamilton, J.L. Stevens, S.M. Foiles, Phys. Rev. Lett. 75 (1995) 4242. 
[12] C. Romainczyk, Ph.D. thesis, Ecole Polytechnique Fédérale, Lausanne, 1994.

[13] T. Härtel, U. Strüber, J. Küppers, Thin Solid Films 229 (1993) 163.

[14] G. Rangelov, T. Fauster, U. Strüber, J. Küppers, Surf. Sci. $331-333$ (1995) 948.

[15] During preparation of the manuscript, we became aware that dislocations in submonolayer $\mathrm{Ag}$ films on $\mathrm{Pt}(111)$ were also observed by S. Gestermann, M. Nohlen, M. Schmidt, K. Wandelt (to be published).

[16] H. Brune, H. Röder, K. Bromann, K. Kern, Thin Solid Films 264 (1995) 230.

[17] C. Ratsch, A.P. Seitsonen, M. Scheffler, Phys. Rev. B 55 (1997) 6750.

[18] H. Röder, E. Hahn, H. Brune, J.P. Bucher, K. Kern, Nature 366 (1993) 141.

[19] Submonolayer coverages of $\mathrm{Ag}$ on $\mathrm{Pt}(111)$ are known to mix into the topmost Pt layer if grown above or annealed to more than $620 \mathrm{~K}$, providing another efficient way of strain relief in this system (see H. Röder, R. Schuster, H. Brune, K. Kern, Phys. Rev. Lett 71 (1993) 2086). However, as alloying is confined to the first atomic layer, complete demixing into separate phases (or no intermixing at all if coverages greater than I ML are annealed) happens as soon as the Ag coverage exceeds $1 \mathrm{ML}$.

[20] R.J. Needs, M.J. Godfrey, M. Mansfield, Surf. Sci. 242 (1991) 215.

[21] M. Bott, M. Hohage, T. Michely, G. Comsa, Phys. Rev. Lett. 70 ( 1993 ) 1489.

[22] A description of the method is given in K.W. Jacobsen, J.K. Nørskov, M.J. Puska, Phys. Rev. B 35 (1987) 7423. The parameters and computer code used can be found in P. Stoltze, J. Phys. Condens. Matter 6 (1994) 9495.

[23] J.A. Meyer, P. Schmid, R.J. Behm, Phys. Rev. Lett. 74 (1995) 3864. 